\title{
BAADE-WESSELINK RADII OF LONG PERIOD CEPHEIDS NEW OBSERVATIONAL RESULTS
}

Iain M. Coulson, John A. R. Caldwell

South African Astronomical observatory

P. O. Box 9 Observatory 7935

\section{Wolfgang Gieren}

Observatorio Astronomico Universidad Nacional

and Physics Department, Universidad de Los Andes, Begota, Colombia

\begin{abstract}
The radii of Galactic Cepheids as determined from a version of the Baade-Wesselink technique are shown to depend upon the colour index used to define the temperature scale. The $(\mathrm{V}-\mathrm{I})$ radii are systematically larger than the commonly used $(B-V)$ radii and are probably better estimators of the true radii. There still remains a problem, however, in reconciling the period-radius relation with the periodluminosity-colour relation.
\end{abstract}

\section{Observations}

A programme of observations of galactic Cepheids with $P>10$ days has recently been completed at SAAO and results will appear in the literature in due course (Coulson \& Caldwell. in preparation). Preliminary results for 9 of these stars are discussed here together with those for 6 other stars, with $9<\mathrm{P}<17$ days (Coulson, Caldwell \& Gieren 1984, in press). These are best compared with similar data for shorter period Cepheids obtained by Gieren (1982, et op cit) and reanalysed here.

Broadband UBVRI photometric measures have been obtained at Sutherland (SAAO) and La Silla (ESO). The measures are on the Cousins' standard system (Menzies, Banfield \& Laing 1980). They have been combined with older photometry from the literature to enable redeterminations of the periods and with the best of this older data to allow full specification of the photometric properties around the pulsation cycle.

The radial velocities were all obtained with the radial velocity spectrometer at the coudé focus of the SAAO $1.9 \mathrm{~m}$ telescope at Sutherland. They are essentially measures of the FeI lines (Coulson 1983) and are on the Wilson (1953) standard system. Older radial velocites in the literature proved to be of considerably poorer quality and have not been combined with the new data.

Phase coverage for the stars discussed here is practically complete and since the photometry and radial velocities have been obtained contemporaneously they allow radius determinations free from the uncertainties of phase mismatching.

\section{Radii}

Radii were obtained using Balona's (1977)maximum-likelihood

version of the Baade-Wesselink method. We have modified his original 
formulation to account for large radial amplitudes $(\delta R / R>0.1)$ and have used not only $V$ and $B-V$ as magnitude and colour, but also $B, R, I$ and $V-R, V-I$ and $R-I$. The derived radii are found to be essentially independent of the choice of magnitude, but show systematic differences with colour. Fig. 1 shows the radius difference

$$
\Delta=\log R_{\mathrm{V}-\mathrm{I}}-\log \mathrm{R}_{\mathrm{B}-\mathrm{V}}
$$

plotted against $\log P$. Clearly $\Delta$ is significantly larger than zero and is probably period dependent.

$$
\begin{gathered}
\Delta=(0.023 \pm 0.016)+(0.029 \pm 0.016) \log \mathrm{P} \\
(\mathrm{se} / \mathrm{pt}=0.023) .
\end{gathered}
$$

If the $(V-I)$ results are less affected by gravity than the (B-V) results then they may be considered better estimates of the sizes of the stars and we may correct ( $B-V$ ) radii by equation ( 1 ). This we have done for the 29 radii derived above plus those for the 32 stars studied by Balona (1977) for which there are reddening estimates (Dean, Warren \& Cousins 1978). These radii yield a mean relation:

$$
\begin{aligned}
& \log R=(0.637 \pm 0.021) \\
& (\text { s.e. } / p t=0.049) .
\end{aligned}
$$

\section{The PLC Zero Point}

With these corrected mean radii we may also determine the mean absolute magnitude

$$
\begin{aligned}
& \left\langle\mathrm{M}_{>}>=A<B-V\right\rangle-5 \log \mathrm{R}+\mathrm{C} \\
& \text { (see Balona } 19 \mathrm{H}_{7} \text {, equation 1.3). }
\end{aligned}
$$

In this equation $C$ is a term usually given as

$$
C=M_{b o 1} \theta+10 \log T_{\text {eff } \theta}-10 a_{0}-b_{0}
$$

were $a_{0}, b_{0}$ are assumed constants of the relations

$$
\begin{aligned}
& \log T_{\text {eff }}=a_{0}+a_{1}\langle B-V\rangle_{0} \\
& M_{b o l}-M_{V}=b_{0}+b_{1}\langle B-V\rangle_{0}
\end{aligned}
$$

From $M y$ we may then derive estimates of the zero-point of the P-L-C relation, $\gamma$ :

$$
\left\langle M_{V}>=\alpha \log P+\beta\langle B-V\rangle_{0}+\gamma\right.
$$

Equations (3) \& (7) yield $\mathrm{C}-\gamma$ in the terms of several observables:

$$
C-\gamma=\alpha \log P+(\beta-A)\langle B-V\rangle_{0}+5 \log R
$$


From the 61 corrected (B-V) - radii derived above we have calculated $C-\gamma$ using nominal values of $\alpha=-3.80, \beta=2.70, A=2.15$ and plot the results against $\log P$ in Figure 2. Cleary $C-\gamma$ is not a constant for all Cepheids. This implies that at least one of the above assumptions is incorrect. It seems most likely that the temperature and/or bolometric correction calibrations may depend upon surface gravity and hence give rise to a period dependence of the type seen here.

Until this dependence is fully quantified the determination of $\gamma$ from Cepheid radil will remain impracticable.

\section{References}

Balona, L.A., 1977. M.N.R.A.S., 178, 231.

Coulson, I.M., 1983. M.N.R.A.S., 200, 925.

Dean, J.F., Warren, P.R. \& Cousins, A.W.J., 1978. M.N.R.A.S., 183, 569. Gieren, W.P., 1982. Ap.J., 260, 208.

Menzies, J.W., Banfield, R.M. \& Laing, J.D., 1980. SAAO Circ. No. 5, p 149.

Wilson, R.E., 1953. General Catalogue of Stellar Radial Velocities, Carnegie Inst. of Washington, D.C.

Figure 1.

Radius Differences

For 29 Galactic Cepheids
Figure 2 .

$C-\gamma$ for 61 Galactic Cepheids using corrected $(B-V)-$ Radil $C-\gamma=-3.8 \log P+0.55\langle B-V\rangle_{0}^{+}$ $5 \log R$.
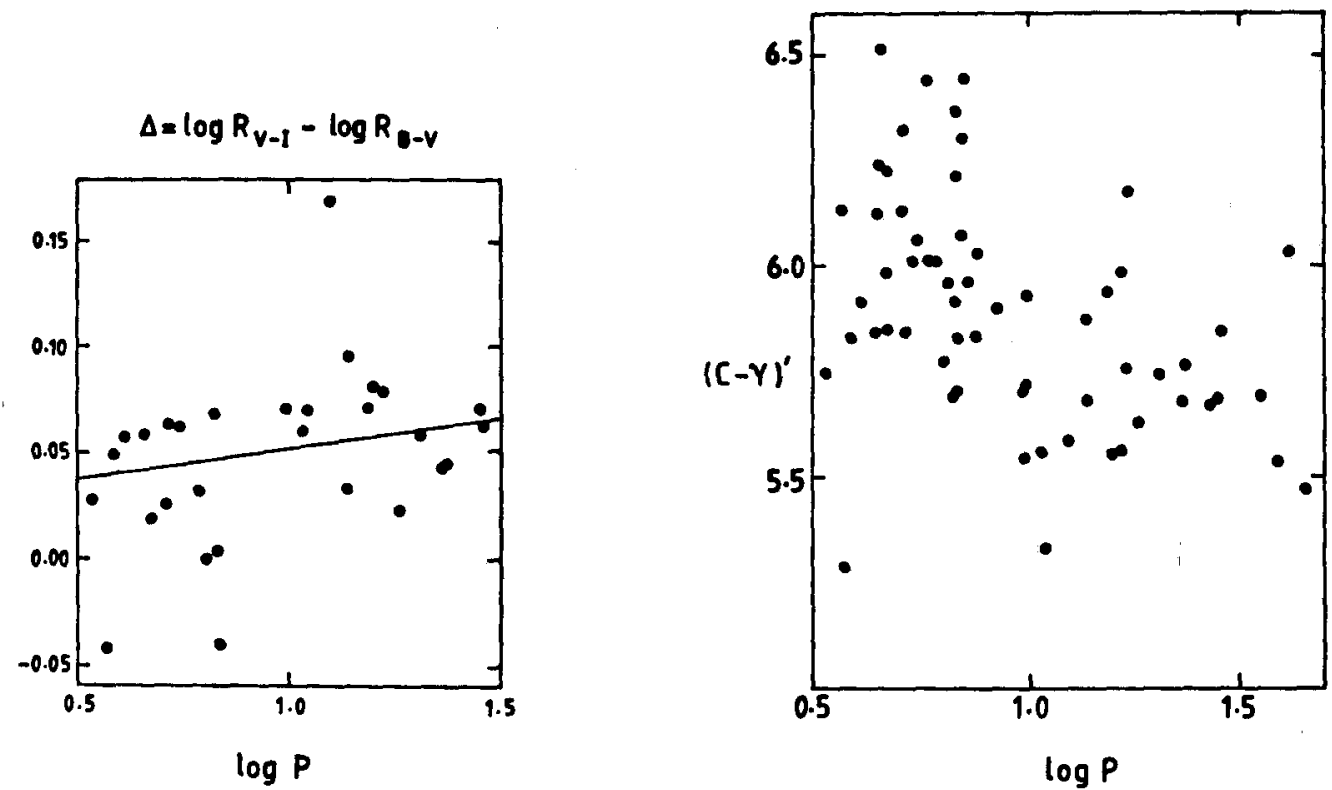